\title{
Effect of the Prandtl number on the onset of turbulence in liquid ${ }^{4} \mathrm{He}$
}

\author{
J. Maurer and A. Libchaber \\ Groupe de Physique des Solides de l'Ecole Normale Supérieure $\left({ }^{*}\right)$, \\ 24, rue Lhomond, 75231 Paris Cedex 05, France
}

(Reçu le 28 juillet 1980, accepté le 16 septembre 1980)

\begin{abstract}
Résumé. - Etude expérimentale de l'évolution des instabilités oscillatoires et du seuil de turbulence dans ${ }^{4} \mathrm{He}$ liquide, en fonction du nombre de Prandtl $(0,4<\operatorname{Pr}<0,8)$ et pour une série de cellules de géométrie réduite comportant deux rouleaux convectifs.

A faible valeur de $\operatorname{Pr}$ le comportement dynamique est similaire à nos études précédentes, à savoir apparition de deux fréquences, suivie d'accrochages et de démultiplication de la basse fréquence, juste avant le seuil de turbulence. A forte valeur de $\operatorname{Pr}$ une nouvelle fréquence apparaît avant le seuil de l'instabilité oscillatoire de Busse. Nous associons ce nouveau régime à un changement de structure des cellules convectives, associé peut-être à la transition skewed varicose prédite par Busse, et qui donne un caractère tridimensionnel à la morphologie des rouleaux.

Différentes bifurcations vers la turbulence sont alors observées. Dans certains cas le seuil de turbulence apparaît avec intermittence temporelle, dans d'autres cas un régime à trois fréquences peut apparaître juste avant le seuil turbulent. Tous ces résultats dépendent fortement du nombre d'onde des rouleaux, ceci en accord avec la théorie de Busse.

Abstract. - We have performed experiments on the onset of turbulence in liquid ${ }^{4} \mathrm{He}$, as a function of the Prandtl number $(0.4<\operatorname{Pr}<0.8)$, for a series of cells of confined geometry with two convective rolls.

For a low value of $\operatorname{Pr}$ the dynamic behaviour is similar to our previous studies, i.e. two frequencies followed by lock-in and subharmonic bifurcations of the lowest frequency, before the onset of turbulence.

For high values of $\operatorname{Pr}$ a new frequency appears before onset of the Busse oscillatory instability. We associate this new regime with a change of the convective roll structure, perhaps due to the skewed varicose instability predicted by Busse, which gives a three dimensional character to the roll geometry.

Various approaches to turbulence are then observed. In some cases the onset of turbulence is intermittent in time; in some others a regime with three frequencies exists before the onset of turbulence. All the results are strongly dependent on the roll wavenumber, in qualitative agreement with the Busse theory.
\end{abstract}

This letter concerns the onset of oscillatory instability and onset of turbulence in a Rayleigh-Bénard experiment in liquid ${ }^{4} \mathrm{He}$. As previously described [1], our experimental cell is a parallelepiped box containing, in the convective state, two rolls perpendicular to the largest lateral side. We report here the effect of a continuous change of the Prandtl number $(0.4<P r<0.8)$. This small variation of the Prandtl number has dramatic effects on the observed onset of instabilities and turbulence. Whereas for a low Prandtl number our results are consistent with our previous publications [1], for a high Prandtl number and a high wavenumber various approaches to turbulence are observed, in agreement in some cases with

(*) Laboratoire associé au Centre National de la Recherche Scientifique.
Gollub and Benson observations in water [2]. One of the approaches leads to a transition to turbulence via intermittency between a quasiperiodic state and a turbulent state.

As shown by Busse [3] and Busse and Clever [4], in this Prandtl number range, two main instabilities are present, the oscillatory instability and the skewed varicose. When the Prandtl number is small, the non linear momentum convection term (the (v. $\nabla) \mathbf{v}$ term) of the equation of motion plays a dominant role. It leads to the oscillatory instability, where a transverse, time dependent, oscillation of the convective rolls propagates along the roll axis. As one increases the Prandtl number, the non linear term in the heat equation, proportional to $(\mathbf{v} . \nabla) T$, can no longer be neglected, and leads to a static instability called by Busse the skewed varicose. It consists of a static periodic thickening and thinning of the convective rolls. In 
liquid ${ }^{4} \mathrm{He}$ those two instabilities are the dominant ones. The stability diagram for convection rolls, as one increases the Rayleigh number, will depend also on the roll wavenumber, as shown on figure 3 of the Busse and Clever [4] paper. For a low wavenumber, the oscillatory instability will appear first as one increases the Rayleigh number. For a higher wavenumber, it depends on the value of the Prandtl number.

Their analysis is relevant to a sample of infinite lateral dimensions, whereas our experimental situation is that of a confined geometry. Nevertheless, we will show that there is qualitative agreement between our experimental study and analysis by Busse, and that the main difference introduced by the lateral boundaries is to increase by about an order of magnitude the value of the Rayleigh number for the onset of instabilities.

1. Experimental situation. - Our experimental setup has been described elsewhere in detail [1]. We use a parallelepiped sample with, in the convective state, two rolls perpendicular to the largest lateral side. For the lateral boundaries [5] we have used various insulators (araldite, nylon, teflon) with thermal conductivities ranging from $10^{-4}$ to $5 \times 10^{-4} \mathrm{~W} /(\mathrm{cm} \mathrm{K})$, the thermal conductivity of the liquid ${ }^{4} \mathrm{He}$ varying in the same range. In the top plate, made out of sapphire, two very small bolometers are inserted. They are cut and machined from an Allen Bradley resistance. Their noise factor has a $1 / f$ overall variation which defines the base line of all our Fourier spectrum analysis (Fig. 2 and Fig. 4). The two bolometers are in contact with the liquid and each placed in front of a convective roll. The temperature signals given by the two bolometers are analysed, using a fast Fourier transform 5420 A H.P. digital analyser. When a time dependent signal is measured, the cross correlation between the two bolometers gives a measure of the phase relation between the oscillating signals. The change in Prandtl number is obtained by varying the temperature in a range from 2.5 to $4.5 \mathrm{~K}$ and the pressure from 1 up to $5 \mathrm{~atm}$. ; we refer to the NBS chart [6] for the calculation of the Prandtl number.

2. Onset of the oscillatory instability. - The Rayleigh number value for the onset of the oscillatory instability is presented in figure 1 as a function of the Prandtl number for three cavities with different aspect ratios. To compare our results with Busse theory, instead of giving an aspect ratio, we define our experimental wavenumber $\alpha$ as $\alpha=\frac{2 \pi \times d}{L}$, where $d$ is the height of the cell and $L$ the largest lateral dimension [7] (which accommodates two convective rolls). The black dots correspond to the onset of the oscillatory instability, defined as follows : the oscillatory instability corresponds to transverse time dependent oscillations of the rolls; thus our bolometers measure an oscillating time dependent signal in phase opposition (when one bolometer is heating the other is cooling).

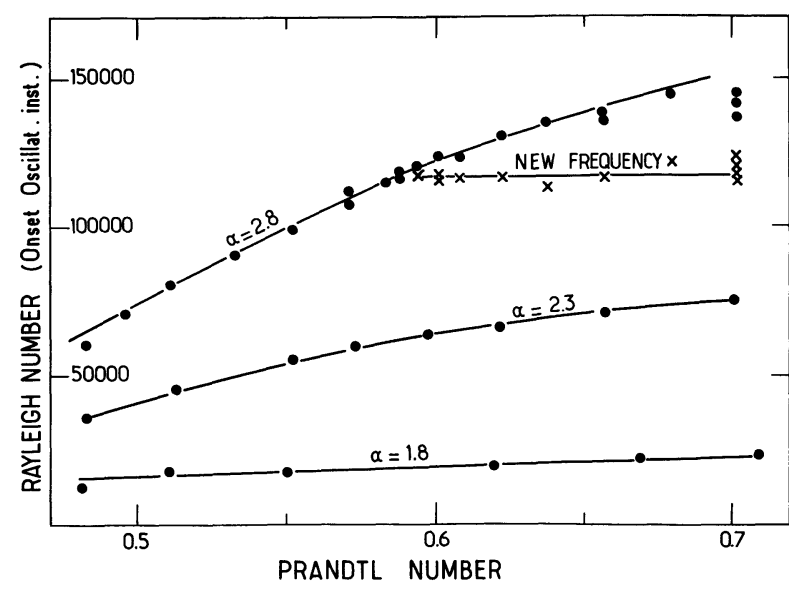

Fig. 1. - The black dots present the evolution, in Rayleigh number, of the onset of the oscillatory instability as a function of the Prandtl number, for three cells of wavenumbers $\alpha=1.8, \alpha=2.3$ and $\alpha=2.8$. The crosses correspond to the onset, in Rayleigh number, of a new time dependent bifurcation.

The frequency of the oscillatory instability for our three cells ranges from $300 \mathrm{mHz}$ to $600 \mathrm{mHz}$, and we will call it $f_{1}$.

If we compare our experimental results with Busse theory, we find the following qualitative agreement : the onset of the instability increases with the Prandtl number. The onset increases also with the roll wavenumber. The main difference, introduced by the lateral boundaries, is that the Rayleigh number for the onset of the instability is increased by about an order of magnitude. The lateral boundaries are thus a stabilizing factor, which is quite reasonable.

For the two cells corresponding to $\alpha=1.8$ and $\alpha=2.3$, the approaches leading to turbulence are as we have described previously [1]. If we keep increasing the Rayleigh number a new bifurcation with a second frequency $f_{2}$ appears, $f_{2}$ being lower than $f_{1}$. For higher Rayleigh numbers, we observe various locking states between $f_{1}$ and $f_{2}$. Just before the onset of turbulence we observe a subharmonic bifurcation of the frequency $f_{2}$. This process has been analysed by Feigenbaum [8] and is in good agreement with our results [1].

For the sample corresponding to $\alpha=2.8$ we observe a striking new phenomena. If $\operatorname{Pr}<0.59$ we have the situation described above. But for $P r \geqslant 0.59$, before the onset of the oscillatory instability we observe another instability characterized as follows : its frequency value is much higher, around $1 \mathrm{~Hz}$, and the phase relation between the signals seen by the bolometers is no longer in phase opposition. In figure 1 it corresponds to the crosses called new frequency. In this regime of high Prandtl number and high wavenumber, the approaches leading to turbulence are more complex and varied. For the cell corresponding to $\alpha=2.8$, the value of this new frequency is about 1.2 Hz. The threshold for this instability does not vary with the Prandtl number. Then, as one increases the Rayleigh number a second frequency sets in $f_{2}$, in 
agreement in frequency value and phase relationship with the oscillatory instability.

Before the onset of turbulence a third frequency is generated, $f_{3}$, first with no locking between the three modes. Then a locking state is measured as shown on figure 2 where $f_{1}-3 f_{2}=f_{3}$. All the peaks in figure 2

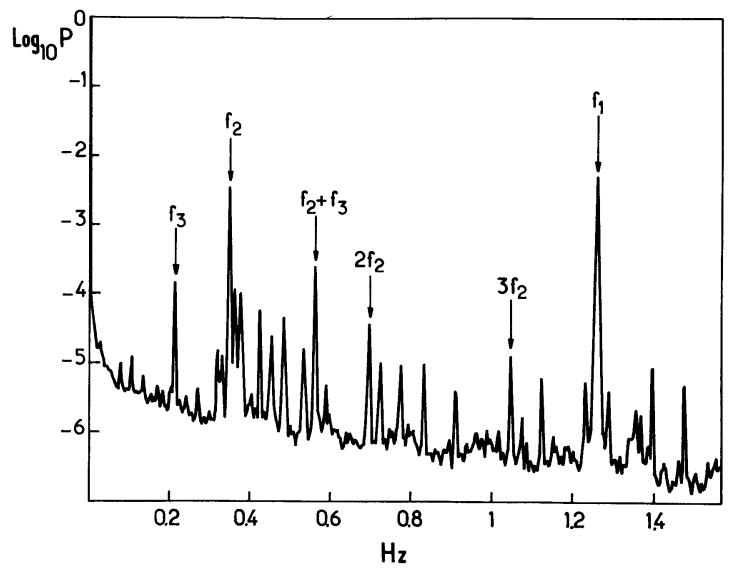

Fig. 2. - Fourier spectrum corresponding to the cell, $\alpha=2.8$, $P r=0.62$, near the onset of turbulence, with three frequencies present, and the locking state $f_{1}-3 f_{2}=f_{3}$. All the peaks correspond to various combinations of the three frequencies (bandwidth $0-1.5 \mathrm{~Hz}$, averaging time $1.35 \times 10^{3} \mathrm{~s}$.)

can be analysed as a combination of the three frequencies. In this recording a weak low frequency noise is already present. This approach to turbulence has been observed in water by Gollub and Benson [2].
Without visualization, it is difficult to define this new state of the fluid. However, if we come back to the Busse and Clever [4] analysis and their figure 3, one can relate this new state to the presence of the skewed varicose instability, which gives a three dimensional character to the roll structure in the static state. The new frequency observed could be related to destabilization of part of a roll.

We have tried various samples with different wavenumbers and have always observed the same phase diagram. It is in this state, with what we believe to be the skewed varicose present, that we have observed for the first time a new approach with an intermittent transition to turbulence.

3. Intermittency in the transition to turbulence. This experiment pertains to a sample made out of teflon with $\alpha=2.7(d=1.29 \mathrm{~mm}, L=3 \mathrm{~mm}$, $l=1.5 \mathrm{~mm}$ ) the Prandtl number being 0.62 . Increasing the Rayleigh number, we measure first a frequency $f_{1}$, close to $1 \mathrm{~Hz}$, then a second frequency $f_{2}$ close to $300 \mathrm{mHz}$ (in phase opposition for the two bolometers). The frequency $f_{1}$ has the same behaviour as the new frequency described in the last chapter and $f_{2}$ relates to the oscillatory instability. The two frequencies are incommensurate and never lock in. The transition to turbulence spreads over an interval of $R / R_{\mathrm{c}}$ from 74.5 to 79 ( $R$ Rayleigh number, $R_{\mathrm{c}}$ Rayleigh number for the onset of convection). The direct time recordings of one bolometer are presented in figure 3, the other

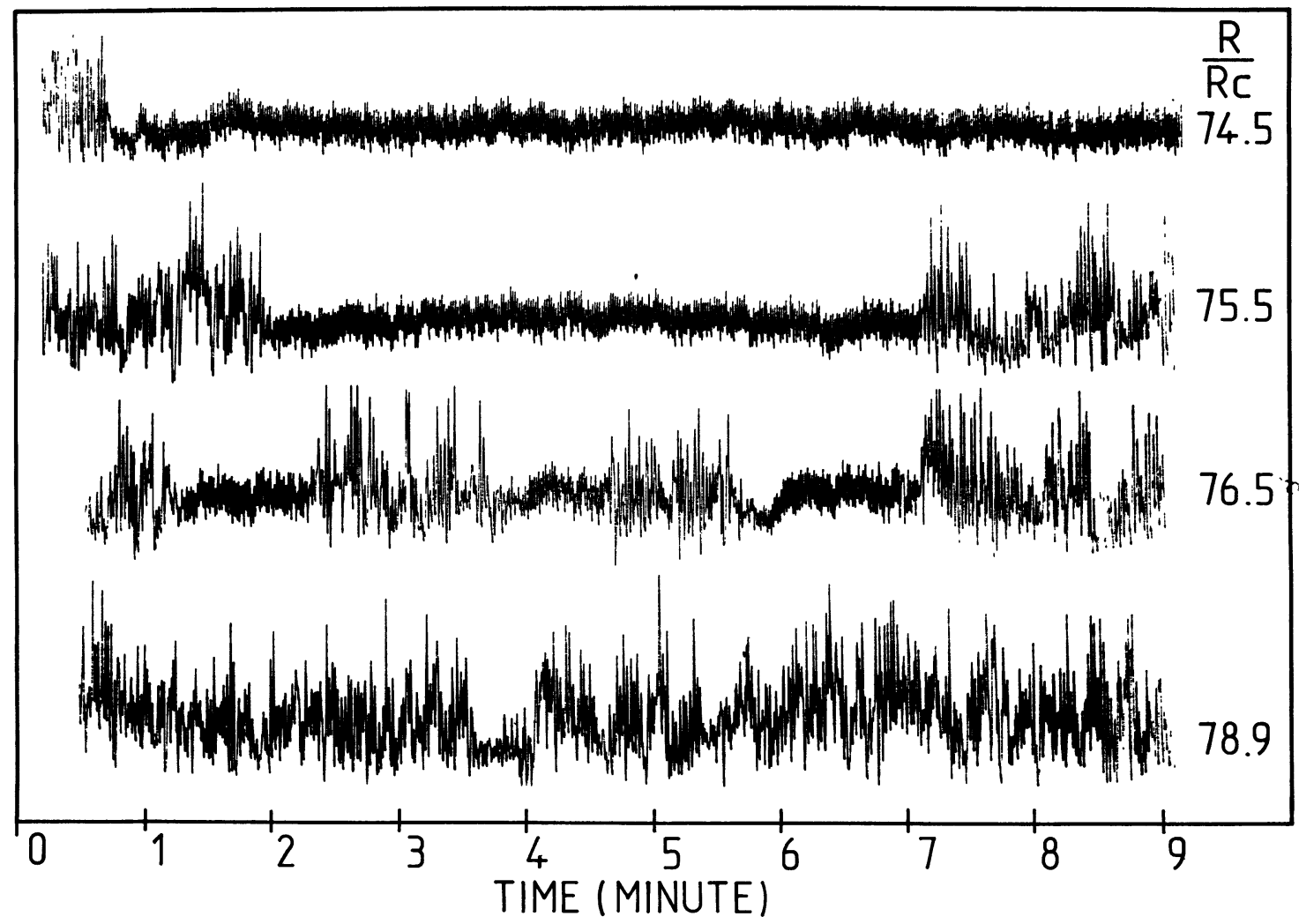

Fig. 3. - Direct time recordings of the intermittent transition to turbulence for a cell, $\alpha=2.7, \operatorname{Pr}=0.62$. ( $R_{\mathrm{c}} \mathrm{Rayleigh}$ number for the onset of convection). For $R / R_{\mathrm{c}}=74.5$, a burst of noise is seen on the left ; otherwise the signal, Fourier analysed, is a combination of two frequencies $f_{1}$ and $f_{2}$ (Fig. 4). 
bolometer presenting the same time recording. If we Fourier analyse the data for $R / R_{\mathrm{c}}=74.5$, we get the spectrum presented on figure 4 . The frequencies $f_{1}$ and $f_{2}$ and their combinations are present, with no lock-in. The peaks are somewhat enlarged due to very

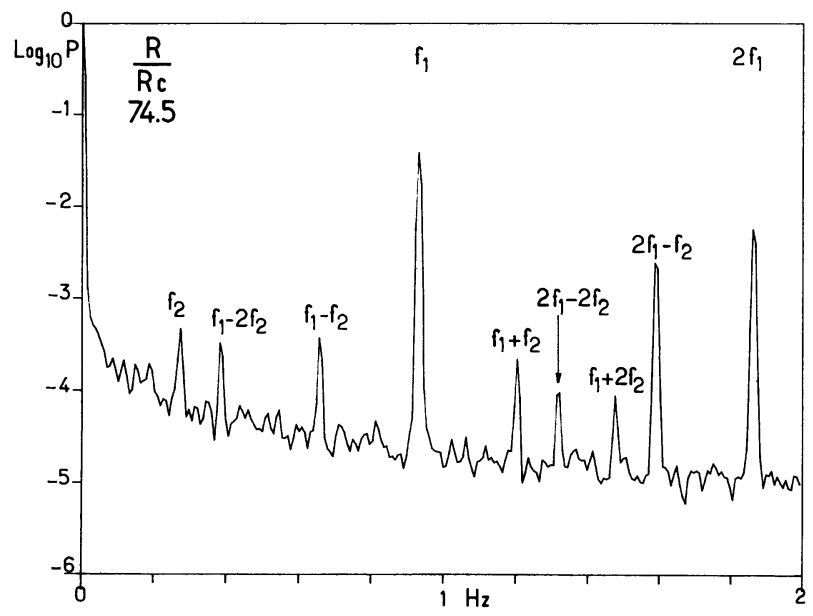

Fig. 4. - Fourier spectrum corresponding to $R / R_{\mathrm{c}}=74.5$ for the cell $\alpha=2.7, \operatorname{Pr}=0.62$ (bandwidth $0-2 \mathrm{~Hz}$, averaging time $10^{3} \mathrm{~s}$ ).

rare intermittent bursts of noise, as can be seen on the left part of the time recordings of figure 3 . For $R / R_{\mathrm{c}}=75.5$, bursts of noise are more frequent, the signal amplitudes being much larger in the noisy regime than in the laminar coherent regime. Finally, for $R / R_{\mathrm{c}}=78.9$, the signals are almost constantly noisy. Both bolometers see the same phenomena with no apparent delay in the generation of noise.

Prompted by our experimental observations, Man- neville and Pomeau [9] have shown that such intermittent behaviour exists for the Lorenz model. In their paper, the duration of the so-called laminar time intervals, where the signal oscillates without noise, diverges as $\left(R-R_{\mathrm{CI}}\right)^{-1 / 2}$, where $R_{\mathrm{CI}}$ represents the onset of the bursts of noise. Our observation of intermittency has a different character. First, we observe a very large increase in the signal amplitude during the noisy time interval. Second, the duration of our laminar coherent time interval does diverge as one gets close to $R / R_{\mathrm{c}}=74.4$ but with a power law closer to $\left(R-R_{\mathrm{CI}}\right)^{-\beta}$ where $1<\beta<1.5$.

Finally, let us note that intermittent transitions to turbulence have been observed recently in water [2] and silicone oil [10].

4. Conclusion. - Our conclusion from our observations in liquid ${ }^{4} \mathrm{He}$ is as follows :

When the Prandtl number and wavenumber are small enough so that the oscillatory instability is the first bifurcation appearing above the onset of convection, the approaches to turbulence are simple and basically follow a Feigenbaum bifurcation scheme with period multiplication of the lowest frequency. In that case the convection pattern is essentially two dimensional before the onset of the oscillatory instability, the convective rolls being cylinders with a translational symmetry along their axis.

For a high Prandtl number, when the wavenumber is large enough so that a static instability such as the skewed varicose sets in first, the roll pattern becomes three dimensional. This dramatically affects the time dependent instabilities and the onset of turbulence; various regimes can then appear. This conclusion applies only to a sample with restricted geometry [11].

\section{References}

[1] Libchaber, A., Maurer, J., J. Physique Colloq. 41 (1980) C3-51.

MAURer, J., LibChaber, A., J. Physique Lett. 40 (1979) L-419 and 39 (1978) L-369.

[2] Gollub, J. P., Benson, S. V., Time dependent instabilities and the transition to turbulent convection (submitted to the J. Fluid Mechanics).

Gollub, J. P., Benson, S. V., Phys. Rev. Lett. 41 (1978) 625.

[3] Busse, F. H., Rep. Prog. Phys. 41 (1978) 1929.

[4] Busse, F. H., Clever, R. M., J. Fluid Mech. 91 (1979) 319.

[5] For the relative importance of the lateral boundaries : KRISHNAMURKI, R., J. Fluid Mech. 60 (1973) 285.

[6] MCCARTHY, R. D., Thermophysical properties of ${ }^{4} \mathrm{He}$, Nat. Bur. Stand. Tech. Note no 631 (1972).
[7] The three cell dimensions are as follows $(d$ height, $L$ longest side dimension). For $\alpha=1.8, d=0.8 \mathrm{~mm}, L=2.8 \mathrm{~mm}$; for $\alpha=2.3, d=1.1 \mathrm{~mm}, L=3 \mathrm{~mm}$, and for $\alpha=2.8$, $d=1.32 \mathrm{~mm}, L=3 \mathrm{~mm}$. For all samples the other lateral dimension is about $l=1.5 \mathrm{~mm}$

[8] Feigendaum, M. J., Phys. Lett. 74A (1979) 375.

Huderman, B. A., Rudnick, J., Phys. Rev. Lett. 45 (1980) 154.

[9] Manneville, P., Pomeau, Y., Different ways to turbulence in dissipative dynamic systems (to be published in Physica $\mathrm{D}$ ).

[10] Berge, P., Dubois, M., Manneville, P., Pomeau, Y., J. Physique Lett. (1980).

[11] For the other case see : Ahlers, G., Walden, R. W., Phys. Rev. Lett. 44 (1980) 445. 